\title{
Functional Outcome After Mechanical Thrombectomy with or without Previous Thrombolysis
}

\author{
Manuel Machado, MD, ${ }^{*}$ Marta Alves, MSc, ${ }^{\dagger} \ddagger$ Alberto Fior, MD, ${ }^{*}$ \\ Isabel Fragata, MD, PhD, ${ }^{* \prime} \ddagger$ Ana Luísa Papoila, $\mathrm{PhD}, \dagger^{\prime} \ddagger$ João Reis, $\mathrm{MD}^{*}{ }^{*}$ and \\ Ana Paiva Nunes, $\mathrm{MD}^{*}$
}

\begin{abstract}
Introduction: Combined intravenous therapy (IVT) and mechanical thrombectomy (MT) is the standard treatment for acute ischemic stroke (AIS) with large vessel occlusion (LVO). However, the use of IVT before MT is recently being questioned. Objectives: To compare patients treated with IVT before MT with those treated with MT alone, in a real-world scenario. Methods: Retrospective analysis of AIS patients with LVO of the anterior circulation who underwent MT, with or without previous IVT, between 2016 and 2018. Results: A total of 524 patients were included (347 submitted to IVT+MT; 177 to MT alone). No differences between groups were found except for a higher time from stroke onset to $\mathrm{CT}$ and to groin puncture in the MT group (297.5 $\mathrm{min}$ vs $115.0 \mathrm{~min}$ and $394.0 \mathrm{~min}$ vs $250.0 \mathrm{~min}$ respectively, $p<0.001$ ). Multivariable analysis showed that age $<75$ years (OR 2.65, 95\% CI 1.71-4.07, $p<0.001$ ), not using antiplatelet therapy (OR 1.93, 95\% CI 1.21-3.08, $p=0.006$ ), low prestroke mRS (OR 4.33, 95\% CI 1.89-9.89, $p<0.001$ ), initial NIHSS (OR 0.89, 95\% CI 0.86-0.93, $p<0.001$ ), absent cerebral edema (OR 7.83, 95\% CI 3.31-18.51, $p<0.001$ ), and mTICI $2 \mathrm{~b} / 3$ (OR 4.56, 95\% CI 2.17-9.59, $p<0.001$ ) were independently associated with good outcome (mRS 0-2). Conclusions: Our findings support the idea that IVT before MT does not influence prognosis, in a real-world setting.

Key Words: Stroke-Thrombectomy-Thrombolysis-Functional Outcome (C) 2020 Elsevier Inc. All rights reserved.
\end{abstract}

\section{Introduction}

Mechanical thrombectomy (MT) has deeply changed the therapeutic approach to acute ischemic stroke in large vessel occlusions (LVO). ${ }^{1-6}$ Combined therapy of intravenous rtPA and MT is currently the standard treatment for patients with LVO in acute ischemic stroke, ${ }^{7}$ however, in all clinical trials only highly selected patients were enrolled, with the great majority of cases in both arms treated with intravenous rtPA.

From the *Unidade Cerebrovascular, Departamento de Neurociências do Centro Hospitalar Universitário Lisboa Central Portugal; +Epidemiology and Statistics Unit, Research Centre, Centro Hospitalar Universitário de Lisboa Central, Lisbon, Portugal; and ¥NOVA Medical School/Faculdade de Ciências Médicas, Lisbon, Portugal.

Received September 22, 2020; revision received November 15, 2020; accepted November 20, 2020.

Corresponding author. E-mail: manuelsmachado@gmail.com.

1052-3057/\$ - see front matter

(c) 2020 Elsevier Inc. All rights reserved.

https:/ / doi.org/10.1016/j.jstrokecerebrovasdis.2020.105495
In a meta-analysis of several randomized trials, the effect of MT was not influenced by intravenous therapy (IVT), raising the question of whether IVT is beneficial in patients with large vessel occlusion. ${ }^{8}$

It is known that IVT has low effectiveness in the recanalization of large vessels. ${ }^{9}$ Additionally, other potential drawbacks of IVT before MT include delayed initiation of MT, major bleeding, neurotoxicity, and disruption of the blood-brain barrier. ${ }^{10-12}$ Nevertheless, administration of IVT can have advantages. Tissue plasminogen activator promotes the breakdown of fibrin polymers. This way, it could favor the success of MT, not only by softening and reducing the clot size, but also by lysing possible distal small emboli and improving overall microvascular reperfusion, optimizing cerebral perfusion distal to the occlusion. ${ }^{12,13}$

Our main goal was to evaluate if patients treated with IVT before MT had a better functional outcome at 90 days after stroke than those treated with MT alone in a real-world scenario. Moreover, we aimed to evaluate if other variables such as age, baseline functional status, neurologic deficits and recanalization rates influence the functional outcome. 


\section{Methods}

All clinical information was retrospectively collected from our prospective acute stroke database. The study protocol was approved by the Ethics Committee of the [Centro Hospitalar Universitário Lisboa Central].

All patients over 18 years old with acute ischemic stroke and LVO of the anterior circulation who underwent MT, with or without previous treatment with IVT, between January 2016 and December 2018, were included. Patients were either referred directly to our hospital (mothership), or transferred from primary stroke centers for MT. Patients with LVO were selected to MT according to international guidelines: time from symptom onset $\leq$ $6 \mathrm{~h}$, Alberta Stroke Program Early CT (ASPECT) score $\geq 6$ and National Institutes of Health Stroke Scale (NIHSS) on admission $\geq 4$, baseline modified Rankin Scale (mRS) score $>2$. In particular cases, the decision to treat with MT resulted from a consensus between the stroke physician and the interventional neuroradiologist, using advanced imaging to evaluate collateral circulation and core-perfusion or core-clinical mismatch.

Primary outcome was mRS score at 90 days after stroke onset. A good outcome was defined as mRS 0-2 at 90 days, and poor outcome was defined as mRS 3-6 at 90 days.

Pre-stroke, stroke, procedural and acute post-stroke characteristics were analyzed to assess their effects on functional outcome. Pre-stroke characteristics were age, sex, medical history (cerebral infarction/transient ischemic attack, hypertension, antiplatelets and anticoagulants treatment, diabetes mellitus, dyslipidemia, current smoker, and atrial fibrillation), prestroke mRS, time from stroke onset to Computed Tomography (CT) Scan and from onset to groin puncture.

Stroke characteristics were NIHSS score, stroke etiology and vessel occlusion location (ICA, MCA and ACA).

Procedural characteristics were type of acute intervention (Endovascular Stroke Treatment alone or IVT+Endovascular Stroke Treatment), and modified Thrombolysis in Cerebral Infarction (mTICI) score.

Acute post-stroke characteristics were cerebral hemorrhage, edema according to the European Cooperative Acute Stroke Study (ECASS II) ${ }^{14}$ and successful revascularization with mTICI score $2 \mathrm{~b}$ and 3 .

The location of vessel occlusion was determined by CT angiogram performed immediately after non contrast head CT. Cerebral hemorrhage and edema were evaluated by CT scan $24 \mathrm{~h}$ after thrombectomy. For cerebral edema, COED 2 (Focal brain swelling up to one third of the hemisphere) or COED 3 (Focal brain swelling greater than one third of the hemisphere Brain swelling with midline shift) were considered. For cerebral hemorrhage $\mathrm{PH} 2$ (hemorrhage exceeding $30 \%$ of the infarct area with significant space occupying effect) and PHr2 (Large confluent hemorrhage in an area remote from the actual infarct; significant space occupying effect may be present) were considered. ${ }^{15}$
Thrombectomy was performed using aspiration (ADAPT) (Penumbra, Alameda, CA) as the first choice, by an experienced interventional neuroradiologist. We performed percutaneous transluminal angioplasty, intra-arterial injection of alteplase, or other procedures depending on the case. The selection of devices/technique was according to the discretion of each operator. Thrombolysis was performed according to standard international guidelines.

\section{Statistical analysis}

Continuous variables were described with median and inter-quantile range (IQR: $\left.\mathrm{P}_{25}-\mathrm{P}_{75}\right)$, and categorical variables as frequencies (percentages).

Nonparametric Chi-Square, Fisher's exact and MannWhitney tests were used, as appropriate.

Univariable and multivariable analyses were performed using logistic regression models. In the multivariable regression analysis, all the variables that had a pvalue $<0.25$ in the univariable analysis were considered. Crude and adjusted odds ratios were estimated (OR) with corresponding 95\% confidence intervals (95\%CI). A sensitivity analysis with propensity scores techniques was performed. The level of significance was $\alpha=0.05$. Data analysis was performed using the Statistical Package for the Social Sciences for Windows, version 25.0 (IBM Corp. Released 2017. IBM SPSS Statistics for Windows, Version 25.0. Armonk, NY: IBM Corp.)

\section{Results}

There were 524 patients in our cohort. Median age was 76 (IQR: 64-83), and 47.1\% were male. Three hundred and forty-seven patients $(66.2 \%)$ were submitted to IVT previous to MT, and 177 to MT alone (33.8\%). There were no statistically significant differences between the two groups at baseline (Table 1) with exception of time from stroke onset to CT scan and to groin puncture, that were higher in the MT only group (297.5 min vs $115.0 \mathrm{~min}$ for time to CT scan, and $394.0 \mathrm{~min}$ vs $250.0 \mathrm{~min}$ for time to groin puncture, $p<0.001$ for both), and previous vascular disease, more prevalent in the MT only group (20.9\% vs $11.5 \%, p=0.004)$.

There was no statistically significant difference in mRS at 90 days between the two groups ( $p=0.759$ ) (Fig. 1).

In the analysis of the excellent outcome (mRS 0-1), 31\% of the patients of the IVT + MT had an excellent outcome at 90 days, when compared to $32 \%$ on the MT only group $(p=0.345)$. There were also no significant differences in death rate at 90 days between the two groups $(17.9 \%$ of patients in IVT+MT vs $23.2 \%$ of patients in MT alone, $p=0.149$ ) (Fig 1).

Table 2 shows the baseline characteristics of patients according to $\mathrm{mRS}$ at 90 days. Two hundred and eighteen patients $(41.6 \%)$ had a good outcome (mRS 0-2) and 306 $(58.4 \%)$ had a poor outcome (mRS 3-6), of which with 103 deaths $(33.7 \%)$. The univariable analysis showed that 
Table 1. Comparison of treatment groups

\begin{tabular}{|c|c|c|c|}
\hline & MT group $(n=177)$ & IVT+MT group $(n=347)$ & $p$-value \\
\hline Age, years, median $\left(\mathrm{P}_{25}-\mathrm{P}_{75}\right)$ & $75(53-81)$ & $76(65-83)$ & 0.330 \\
\hline Male, $\mathrm{n}(\%)$ & $89(50.3 \%)$ & $158(45.5 \%)$ & 0.303 \\
\hline \multicolumn{4}{|l|}{ Co-morbidities, n (\%) } \\
\hline $\mathrm{AF}$ & $61(34.5 \%)$ & $139(40.1 \%)$ & 0.212 \\
\hline Antiplatelet therapy & $60(33.9 \%)$ & $103(29.7 \%)$ & 0.285 \\
\hline DM & $37(20.9 \%)$ & $74(21.3 \%)$ & 0.916 \\
\hline HTA & $135(76.3 \%)$ & $260(74.9 \%)$ & 0.729 \\
\hline Dyslipidemia & $90(50.8 \%)$ & $147(42.4 \%)$ & 0.065 \\
\hline Previous stroke & $22(12.4 \%)$ & $31(8.9 \%)$ & 0.112 \\
\hline TIA & $2(1.1 \%)$ & $8(2.3 \%)$ & 0.270 \\
\hline Anticoagulation & $9(5.1 \%)$ & $22(6.3 \%)$ & 0.564 \\
\hline Vascular Disease & $37(20.9 \%)$ & $40(11.5 \%)$ & 0.004 \\
\hline Previous mRS 0-2, n (\%) & $151(85.3 \%)$ & $312(89.9 \%)$ & 0.091 \\
\hline NIHSS at entrance, median $\left(\mathrm{P}_{25}-\mathrm{P}_{75}\right)$ & $16(11-22)$ & $17(12-22)$ & 0.566 \\
\hline \multicolumn{4}{|l|}{ Time from onset } \\
\hline to $\mathrm{CT}$ scan, min, median $\left(\mathrm{P}_{25}-\mathrm{P}_{75}\right)$ & $297.5(140.5-466.5)$ & $115.0(90.0-171.5)$ & $<0.001$ \\
\hline to groin puncture, min, median $\left(\mathrm{P}_{25}-\mathrm{P}_{75}\right)$ & $394.0(255.5-605.0)$ & $250.0(180.0-328.0)$ & $<0.001$ \\
\hline Transfer, n (\%) & $93(52.5 \%)$ & $153(44.1 \%)$ & 0.066 \\
\hline mTICI score $2 b-3, n(\%)$ & $155(87.6 \%)$ & $295(85.0 \%)$ & 0.434 \\
\hline Haemorrhage (PH2 or PHr2), n (\%) & $10(5.6 \%)$ & $11(3.2 \%)$ & 0.097 \\
\hline Oedema (COED 2 or 3$), \mathrm{n}(\%)$ & $30(16.9 \%)$ & $44(12.7 \%)$ & 0.143 \\
\hline NIHSS $24 \mathrm{H}$, median $\left(\mathrm{P}_{25}-\mathrm{P}_{75}\right)$ & $13(4-21)$ & $11(4-20)$ & 0.686 \\
\hline mRS $0-2$ at 90 days, $\mathrm{n}(\%)$ & $72(40.7 \%)$ & $146(42.1 \%)$ & 0.759 \\
\hline
\end{tabular}

AF - Atrial fibrillation; CT - Computed tomography; DM - Diabetes Mellitus; HTA - hypertension; IVT - Intravenous therapy; mRS modified Rankin Scale; MT - Mechanical thrombectomy; mTICI - modified Thrombolysis in Cerebral Infarction; NIHSS - National Institutes of Health Stroke Scale; TIA - Transient Ischemic Attack.

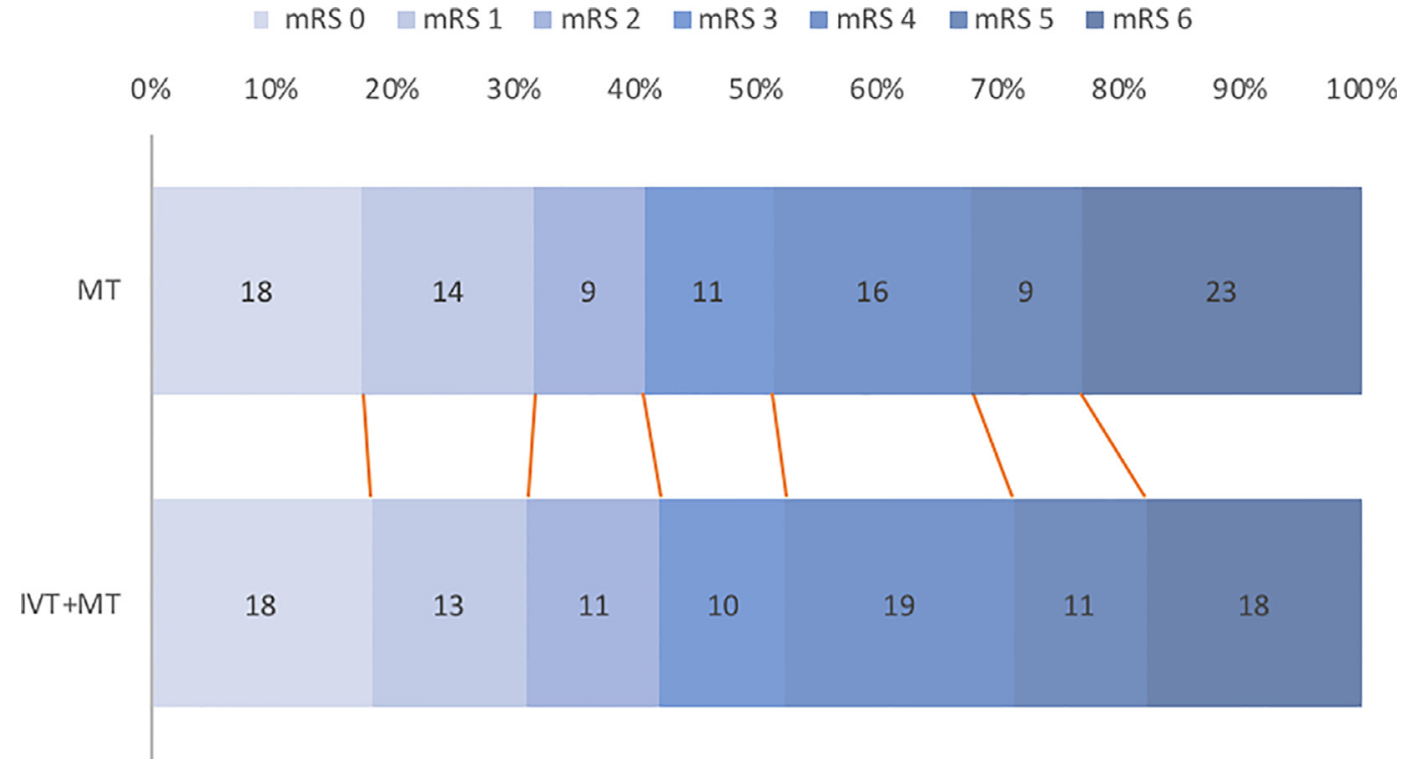

Fig. 1. Distribution of modified Ranking Scale (mRS) at 90 days depending on treatment modality.

lower age (median 72 versus 78 years, $p<0.001$ ), male sex (52.8\% versus $43.1 \%, p=0.030)$, low $(0-2)$ prestroke $\mathrm{mRS}$ (95.4\% versus $83.3 \%, p<0.001)$, lower initial NIHSS score (median 14 versus $19, p<0.001)$, occlusion site M1 $(59.2 \%$ versus $53.6 \%, p=0.031$ ), and occlusion site $\mathrm{M} 2+\mathrm{M} 3+\mathrm{ACA}$ $(19.7 \%$ versus $16.0 \%, p=0.038)$, mTICI score $2 \mathrm{~b}$ or 3
$(91.3 \%$ versus $77.8 \%, p<0.001)$ and NIHSS score at $24 \mathrm{~h}$ after stroke (median 4 versus 18, $p<0.001$ ) were associated with a good outcome. On the other hand, medical history of atrial fibrillation $(29.4 \%$ versus $42.5 \%$, $p=0.002)$, antiplatelet therapy $(24.8 \%$ versus $35.6 \%$, $p=0.008)$, hypertension $(68.3 \%$ versus $79.7 \%, p=0.003)$, 
Table 2. Baseline clinical characteristics of patients and $m R S$ at 90 days.

\begin{tabular}{|c|c|c|c|c|}
\hline & All $(n=524)$ & mRS $0-2(n=218)$ & $m R S \geq 3(n=306)$ & $\mathrm{p}$-value \\
\hline Age, years, median $\left(\mathrm{P}_{25}-\mathrm{P}_{75}\right)$ & $76(64-83)$ & $72(57-79)$ & $78(69-84)$ & $<0.001$ \\
\hline Male, n (\%) & $247(47.1 \%)$ & $115(52.8 \%)$ & $132(43.1 \%)$ & 0.030 \\
\hline \multicolumn{5}{|l|}{ Medical record, n (\%) } \\
\hline $\mathrm{AF}$ & $194(37.0 \%)$ & $64(29.4 \%)$ & $130(42.5 \%)$ & 0.002 \\
\hline Antiplatelet therapy & $163(31.1 \%)$ & $54(24.8 \%)$ & $109(35.6 \%)$ & 0.008 \\
\hline $\mathrm{DM}$ & $111(21.2 \%)$ & $41(18.8 \%)$ & $70(22.9 \%)$ & 0.262 \\
\hline HTA & $393(75.0 \%)$ & $149(68.3 \%)$ & $244(79.7 \%)$ & 0.003 \\
\hline Dyslipidemia & $237(45.2 \%)$ & $90(41.3 \%)$ & $147(48.0 \%)$ & 0.126 \\
\hline Previous stroke & $53(10.1 \%)$ & $16(07.3 \%)$ & $37(12.0 \%)$ & 0.078 \\
\hline TIA & $10(1.9 \%)$ & $1(0.4 \%)$ & $9(3.0 \%)$ & 0.075 \\
\hline Anticoagulation & $31(5.9 \%)$ & $11(5.0 \%)$ & $20(6.5 \%)$ & 0.477 \\
\hline Vascular Disease & $77(14.7 \%)$ & $28(12.9 \%)$ & $49(16.0 \%)$ & 0.313 \\
\hline Previous mRS 0-2, n (\%) & $463(88.4 \%)$ & $208(95.4 \%)$ & $255(83.3 \%)$ & $<0.001$ \\
\hline \multicolumn{5}{|l|}{ Etiology, $\mathrm{n}(\%)$} \\
\hline Large vessel & $67(12.7 \%)$ & $31(14.2 \%)$ & $36(11.8 \%)$ & 0.450 \\
\hline Embolic & $237(45.2 \%)$ & $78(35.8 \%)$ & $159(52.0 \%)$ & $<0.001$ \\
\hline Not & $188(35.9 \%)$ & $86(39.5 \%)$ & $102(33.3 \%)$ & \\
\hline Unusual & $32(6.1 \%)$ & $23(10.5 \%)$ & $9(2.9 \%)$ & 0.639 \\
\hline NIHSS at entrance, median $\left(\mathrm{P}_{25}-\mathrm{P}_{75}\right)$ & $16(12-22)$ & $14(8-19)$ & $19(15-23)$ & $<0.001$ \\
\hline Patients with previous IVT, n (\%) & $347(66.2 \%)$ & $146(67.0 \%)$ & $201(65.7 \%)$ & 0.759 \\
\hline \multicolumn{5}{|l|}{ Time from onset } \\
\hline to $\mathrm{CT}$ scan, min, median $\left(\mathrm{P}_{25}-\mathrm{P}_{75}\right)$ & $139.5(92.0-233.3)$ & $146.5(90.0-248.8)$ & $137.0(97.5-222.5)$ & 0.475 \\
\hline to groin puncture, min, median $\left(\mathrm{P}_{25}-\mathrm{P}_{75}\right)$ & $282.0(197.0-384.5)$ & $265.0(189.0-384.0)$ & $290.5(201.8-375.0)$ & 0.731 \\
\hline \multicolumn{5}{|l|}{ Occlusion site, $\mathrm{n}(\%)$} \\
\hline ICA & $139(26.5 \%)$ & $46(21.1 \%)$ & $93(30.4 \%)$ & 0.550 \\
\hline M1 & $293(55.9 \%)$ & $129(59.2 \%)$ & $164(53.6 \%)$ & 0.031 \\
\hline $\mathrm{M} 2+\mathrm{M} 3+\mathrm{ACA}$ & $92(17.6 \%)$ & $43(19.7 \%)$ & $49(16.0 \%)$ & 0.038 \\
\hline Patients with no bridging, $\mathrm{n}(\%)$ & $246(47.0 \%)$ & $108(49.5 \%)$ & $138(45.1 \%)$ & 0.315 \\
\hline mTICI score $2 \mathrm{~b}-3, \mathrm{n}(\%)$ & $437(83.4 \%)$ & $199(91.3 \%)$ & $238(77.8 \%)$ & $<0.001$ \\
\hline Haemorrhage (PH2 or $\mathrm{PHr} 2), \mathrm{n}(\%)$ & $21(4.0 \%)$ & $2(0.9 \%)$ & $19(6.2 \%)$ & 0.009 \\
\hline Oedema (COED 2 or 3$), \mathrm{n}(\%)$ & $74(14.1 \%)$ & $7(3.2 \%)$ & $67(21.9 \%)$ & $<0.001$ \\
\hline NIHSS $24 \mathrm{H}$, median $\left(\mathrm{P}_{25}-\mathrm{P}_{75}\right)$ & $11(4-20)$ & $4(1-8)$ & $18(12-23)$ & $<0.001$ \\
\hline
\end{tabular}

ACA - anterior cerebral artery; AF - Atrial fibrillation; CT - Computed tomography; DM - diabetes mellitus; HTA - hypertension; ICA Internal Carotid Artery; IVT - intravenous therapy; mRS - modified Rankin Scale; MT - Mechanical thrombectomy; mTICI - modified Thrombolysis In Cerebral Infarction; NIHSS - National Institutes of Health Stroke Scale; TIA - Transient Ischemic Attack. p-values were obtained by univariable logistic regression models.

embolic stroke etiology (35.8\% versus $52.0 \%, p<0.001)$, large hemorrhage complication $(0.9 \%$ versus $6.2 \%$, $\mathrm{p}=0.009)$ and significant edema $(3.2 \%$ versus $21.9 \%$, $p<0.001)$ were associated with a poor outcome.

The multivariable analysis showed that age under 75 years (OR 2.65, 95\% CI 1.71-4.07, $p<0.001$ ), not using antiplatelet therapy (OR 1.93, 95\% CI 1.21-3.08, $p=0.006)$, low prestroke mRS (0-2) (OR 4.33, 95\% CI $1.89-9.89, p<0.001$ ), initial NIHSS score (OR 0.89, 95\% CI $0.86-0.93, p<0.001$ ), no significant edema (OR 7.83, 95\% CI 3.31-18.51, $p<0.001$ ), and mTICI $2 \mathrm{~b}$ or 3 (OR $4.56,95 \%$ CI 2.17-9.59, $p<0.001$ ) was independently associated with a good outcome.

From the univariable analysis, all the above variables were selected for the multivariable study, but only younger age, previous antiplatelet therapy, low prestroke mRS, low initial NIHSS score, no significant edema, and mTICI $\geq 2 b$ remained independently associated with a good outcome.
Accordingly, age under 75 years old had almost a three-fold increase in the odds of a good outcome (OR 2.65, 95\% CI $1.71-4.07, p<0.001)$. Patients not using antiplatelet therapy and not having significant edema had approximately a twofold (OR 1.93, 95\% CI 1.21-3.08, $p=0.006$ ) and an eight-fold (OR 7.83, 95\% CI 3.31-18.51, $p<0.001$ ) increase in the odds of a good outcome, respectively. For each increase of 1 unit on the initial NIHSS score, there was an $11 \%$ reduction in the odds of having a good outcome (OR 0.89, 95\% CI $0.86-0.93, p<0.001)$. Also, a prestroke mRS (0-2) and patients with TICI $2 \mathrm{~b}$ or 3 had approximately a four-fold (OR 4.33, 95\% CI 1.89-9.89, $p<0.001$, and OR 4.56, 95\% CI $2.17-9.59, p<0.001$, respectively) increase in the odds of a good outcome. Treatment group was not chosen to be part of the final regression model.

The results obtained by the propensity scores methods were similar as no difference between the two treatment groups was observed. 


\section{Discussion}

We compared patients with acute stroke due to anterior circulation LVO, submitted either to IVT followed by MT or MT alone in a real-world setting, to evaluate differences in the functional outcome. We found similar results for the two groups in terms of the outcome at 90 days.

The wide adoption of IVT has changed the therapeutic approach of acute ischemic stroke. Although IVT is safe and effective in reducing disability in patients with acute ischemic stroke, it has poor efficacy in the recanalization of large vessels. ${ }^{9}$ More recently, MT has been proven a safe and effective therapy for patients with LVO. The usefulness of endovascular MT for acute ischemic stroke in LVO has shown in several randomized controlled trials to improve the functional outcome and for this reason, combined therapy with MT and IVT is currently the standard of care treatment for patients with acute ischemic stroke with LVO in the anterior circulation. ${ }^{3-8}$

The administration of IV tPA before MT is thought to facilitate clot detachment from the vessel wall as a result of tPA-induced fibrin degradation, and downstream microvascular thrombosis reduction. ${ }^{16}$ Nevertheless, considering that 1) LVO are characterized by a low recanalization rate after IVT; 2) MT has high recanalization rates, and 3) there are concerns of increased intracranial and extracranial bleeding risk with IVT pretreatment, the utility of IVT before MT in LVO has recently been questioned.

It is still unclear whether pretreatment with IVT provides any additional benefits to patients with LVO undergoing MT. ${ }^{17}$ Although there are some studies which showed that combined therapy (IVT+MT) has better outcomes compared with MT alone, ${ }^{18-21}$ there are others in contradiction suggesting no benefit of IVT pretreatment to MT. ${ }^{22-24}$

In our study, the use of IVT previous to MT was not associated with better outcome at 90 days or better NIHSS at $24 \mathrm{H}$ after stroke, when compared to the use of MT alone. These results are in line with previous meta-analyses that found no significant differences in functional outcome after mechanical thrombectomy alone versus combined therapy with previous IVT. ${ }^{25,26}$ Also, a recently published trial comparing endovascular thrombectomy with or without intravenous Alteplase in acute stroke showed no benefit in the use of thrombolysis previous to MT regarding functional outcome. ${ }^{27}$ Other recent papers also found that MT alone or IVT+MT did not differ significantly in functional independence rate at 90 days or median discharge National Institutes of Health Stroke Scale (NIHSS) score. ${ }^{28-31}$

The association between reperfusion success and rate of good outcome was clearly demonstrated in our population, and as with previous studies, our results suggest mTICI to be an important independent predictor of outcome. ${ }^{32-35}$ Our results showed a high reperfusion success rate $(83,4 \%)$ compared with other studies. ${ }^{36-38}$ One of the variables known to influence the recanalization success is the clot composition. Rich red blood cell (RBC) thrombi were positively associated with successful reperfusion after thrombectomy, as opposed to thrombi with low RBC content and high fibrin content. ${ }^{39,40}$ We did not analyse the composition of clots in our sample, which constitutes a limitation. The fact that we used newer aspiration devices might also contribute to our better recanalization rates. $^{41}$

Since the previous administration of IVT can theoretically influence the recanalization rate, we would expect to see a higher recanalization rate in patients that underwent combined treatment with IVT and MT. However we found no differences between groups (IVT+MT and MT alone) in recanalization rates. Although there is some suggestion that the use of IVT may actually reduce the clot size $^{42}$ the evidence that IVT previous to MT actually leads to better recanalization rates is still weak. ${ }^{26,37,42,43}$

In our cohort, we had longer total times from onset to groin puncture in MT alone group when compared to other observational studies. ${ }^{43}$ The time from onset to CT or to groin puncture were the variables with the most relevant differences between groups. We are aware that the longer $\mathrm{CT}$ to groin puncture times in the MT alone group was in many cases probably the reason for not using IVT, and that this fact constitutes a bias in the interpretation of the present data. We could think that the longer CT to groin puncture times in the MT alone group could represent a theoretical advantage in the outcome for the IVT +MT group, however, our analysis does not confirm this. Moreover, the time variable does not seem to be an independent predictor of outcome in these patients. These findings are in line with the evolving idea that shifts from the "time window paradigm" to the "tissue window paradigm". ${ }^{44}$ Two clinical trials ${ }^{45,46}$ (DAWN and DEFUSE-3) showed benefit in patients treated up to 24 hours from symptom onset, disrupting the "time window paradigm". It is now demonstrated that other factors contribute to the degree of ischemic injury at any point in time. ${ }^{47,48}$ The growth of ischemic lesions is of vital importance and this growth varies substantially among patients. Thus, the role of collateral circulation in selecting patients for MT should not be underestimated.

Although the time to endovascular treatment and the use of previous IVT does not seem to influence the outcome, other variables were independently associated with a better outcome at 90 days in our cohort. Favorable prestroke mRS (0-2), lower NIHSS scores at admission, and age under 75 years old were strongly associated with good outcome, suggesting that these variables have a strong influence on the prognosis of patients after MT regardless of the effect of treatment. This is in line with the literature, showing a complex interaction between several other variables, besides time, such as infarct volume, infarct location laterality, age and baseline functional status of the outcome prognosis. ${ }^{49}$ 
Our study has several limitations, mainly its retrospective nature, being a single-center study, with lack of external validation. Also, variables such as ASPECTS were not considered since they were unavailable in our prospective database. This variable seems to play an important role in the outcome in patients with ischemic stroke. Not analyzing it represents a major limitation to the study. Nevertheless, the results are representative of a tertiary stroke center, and of a real world scenario of acute stroke due to LVO.

\section{Conclusion}

In our cohort of patients with LVO that were submitted to MT, younger age, low mRS at baseline, lower NIHSS at presentation, and good reperfusion status after MT were independent predictors of good clinical outcome. The use of previous IVT and time from onset to treatment did not influence outcome. These findings in a real-world scenario support the existing data that IVT does not influence prognosis in patients with anterior circulation LVO, submitted to MT.

\section{References}

1. Berkhemer OA, Fransen PSS, Beumer D, et al. A randomized trial of intraarterial treatment for acute ischemic stroke. N Engl J Med 2015;372(1):11-20. https:/ / doi.org/ 10.1056/NEJMoa1411587.

2. Goyal M, Demchuk AM, Menon BK, et al. Randomized assessment of rapid endovascular treatment of ischemic stroke. N Engl J Med 2015;372(11):1019-1030. https:// doi.org/10.1056/NEJMoa1414905.

3. Campbell BC, Mitchell PJ, Kleinig TJ, et al. Endovascular therapy for ischemic stroke with perfusion-imaging selection. N Engl J Med 2015;372(11):1009-1018. https:// doi. org/10.1056/NEJMoa1414792.

4. Saver JL, Goyal M, Bonafe A, et al. Stent-retriever thrombectomy after intravenous t-PA vs. t-PA alone in stroke. N Engl Med 2015;372(24):2285-2295. https://doi.org/ 10.1056/NEJMoa1415061.

5. Jovin TG, Chamorro A, Cobo E, et al. Thrombectomy within 8 hours after symptom onset in ischemic stroke. N Engl J Med 2015;372(24):2296-2306. https://doi.org/ 10.1056/NEJMoa1503780.

6. Bracard S, Ducrocq X, Mas JL. Mechanical thrombectomy after intravenous alteplase versus alteplase alone after stroke (THRACE): a randomized controlled trial. Lancet Neurol 2016;15(11):1138-1147. https://doi.org/10.1016/ S1474-4422(16)30177-6.

7. Powers WJ, Derdeyn CP, Biller J, et al. 2015 American Heart Association/American Stroke Association Focused Update of the 2013 guidelines for the early management of patients with acute ischemic stroke regarding endovascular treatment: a guideline for healthcare professionals from the American heart association/ American stroke association. Stroke 2015;46:3020-3035. https://doi.org/ 10.1161/STR.0000000000000074.

8. Goyal M, Menon BK, van Zwam WH, et al. Endovascular thrombectomy after large-vessel ischaemic stroke: a meta-analysis of individual patient data from five randomised trials. Lancet 2016;387:1723-1731. https: / / doi.org/ 10.1016/S0140-6736(16)00163-X.

9. Rai A, Cline B, Williams E, et al. Intravenous thrombolysis outcomes in patients presenting with large vessel acute ischemic strokes-CT angiography-based prognosis. J Neuroimaging 2015;25(2):238-242. https:/ / doi.org/ 10.1111/jon.12126.

10. Kidwell CS, Latour L, Saver JL, et al. Thrombolytic toxicity: blood brain barrier disruption in human ischemic stroke. Cerebrovasc Dis 2008;25:338-343. https://doi. org /10.1159/000118379.

11. Fischer U, Kaesmacher J, Mendes Pereira V, et al. Direct mechanical thrombectomy versus combined intravenous and mechanical thrombectomy in large-artery anterior circulation stroke: a topical review. Stroke 2017;48:2912-2918. https:/ / doi.org/10.1161/STROKEAHA.117.017208.

12. Chandra RV, Leslie-Mazwi TM, Mehta BP, et al. Does the use of IV tPA in the current era of rapid and predictable recanalization by mechanical embolectomy represent good value? J Neurointerv Surg 2016;8:443-446. https: / / doi.org/10.1136/neurintsurg-2015-012231.

13. Desilles J-P, Loyau S, Syvannarath V, et al. Alteplase reduces downstream microvascular thrombosis and improves the benefit of large artery recanalization in stroke. Stroke 2015;46:3241-3248. https://doi.org/ 10.1161/STROKEAHA.115.010721.

14. Hacke W, Kaste M, Fieschi C, et al. Randomised doubleblind placebo-controlled trial of thrombolytic therapy with intravenous alteplase in acute ischaemic stroke (ECASS II). Second European-Australasian acute stroke study investigators. Lancet 1998;352(9136):1245-1251. https: / / doi.org/10.1016/s0140-6736(98)08020-9.

15. The SITS open artery by thrombectomy in acute occlusive stroke study (SITS Open). SITS Open protocol version 5.0 2016; 1-83.

16. Fischer U, Kaesmacher J, Molina CA, et al. Primary thrombectomy in tPA (tissue-type plasminogen activator) eligible stroke patients with proximal intracranial occlusions. Stroke 2018;49(1):265-269. https://doi.org/ 10.1161/STROKEAHA.117.018564.

17. Leslie-Mazwi T, Chandra RV, Baxter BW, et al. ELVO: an operational definition. J Neurointerv Surg 2018;10(6):507509. https: / / doi.org/10.1136/neurintsurg-2018-013792.

18. Goyal N, Tsivgoulis G, Frei D, et al. Comparative safety and efficacy of combined IVT and MT with direct MT in large vessel occlusion. Neurology 2018;90(15):e1274-e1282. https:/ / doi.org/10.1212/WNL.0000000000005299.

19. Mistry EA, Mistry AM, Nakawah MO, et al. Mechanical thrombectomy outcomes with and without intravenous thrombolysis in stroke patients: a meta-analysis. Stroke 2017;48(9):2450-2456. https://doi.org/10.1161/STROKEAHA.117.017320.

20. Di Maria F, Mazighi M, Kyheng M, et al. Intravenous thrombolysis prior to mechanical thrombectomy in acute ischemic stroke: silver bullet or useless bystander? J Stroke 2018;20(3):385-393. https://doi.org/10.5853/ jos.2018.01543.

21. Goyal N, Tsivgoulis G, Pandhi A, et al. Impact of pretreatment with intravenous thrombolysis on reperfusion status in acute strokes treated with mechanical thrombectomy. J Neuro Surg 2019;11:1073-1079. https://doi.org/ $10.5853 /$ jos.2018.01543.

22. Broeg-Morvay A, Mordasini P, Bernasconi C, et al. Direct mechanical intervention versus combined intravenous and mechanical intervention in large artery anterior circulation stroke: a matched-pairs analysis. Stroke 2016;47(4):10371044. https:/ / doi.org/10.1161/STROKEAHA.115.011134.

23. Bellwald S, Weber R, Dobrocky T, et al. Direct mechanical intervention versus bridging therapy in stroke patients eligible for intravenous thrombolysis: a pooled analysis 
of 2 registries. Stroke 2017;48(12):3282-3288. https:/ / doi. org/10.1161/STROKEAHA.117.018459.

24. Coutinho JM, Liebeskind DS, Slater LA, et al. Combined intravenous thrombolysis and thrombectomy vs thrombectomy alone for acute ischemic stroke: a pooled analysis of the SWIFT and STAR studies. JAMA Neurol 2017;74(3):268274. https://doi.org/10.1001/jamaneurol.2016.5374.

25. Kim CH, Jeon JP, Kim SE, et al. Endovascular treatment with intravenous thrombolysis versus endovascular treatment alone for acute anterior circulation stroke: a meta-analysis of observational studies. J Korean Neurosurg Soc 2018;61(4):467-473. https://doi.org/10.3340/ jkns.2017.0505.006.

26. Phan K, Dmytriw AA, Maingard J, et al. Endovascular thrombectomy alone versus combined with intravenous thrombolysis. World Neurosurg 2017;108:850-858.e2. https:/ / doi.org/10.1016/j.wneu.2017.08.040.

27. Yang P, Zhang Y, Zhang L, et al. Endovascular thrombectomy with or without intravenous alteplase in acute stroke. N Engl J Med 2020;382(21):1981-1993. https:// doi.org/10.1056/NEJMoa2001123.

28. Zha M, Huang K, Yang D, et al. Bridge mechanical thrombectomy may be a better choice for acute large vessel occlusions [published online ahead of print, 2020 Oct 20]. J Thromb Thrombolysis 2020. https://doi.org/10.1007/ s11239-020-02307-0.

29. Hassan AE, Kotta H, Garza L, et al. Pre-thrombectomy intravenous thrombolytics are associated with increased hospital bills without improved outcomes compared with mechanical thrombectomy alone. J Neurointerv Surg 2019;11(12):1187-1190. https://doi.org/10.1136/ neurintsurg-2019-014837.

30. Kurminas M, Berūkštis A, Misonis N, et al. Intravenous rtPA dose influence on outcome after middle cerebral artery ischemic stroke treatment by mechanical thrombectomy. Medicina (Kaunas) 2020;56(7):357. https:/ / doi. org /10.3390/medicina56070357.

31. Hassan AE, Ringheanu VM, Preston L, et al. IV tPA is associated with increase in rates of intracerebral hemorrhage and length of stay in patients with acute stroke treated with endovascular treatment within 4.5 hours: should we bypass IV tPA in large vessel occlusion? J Neuro Surg 2020. https://doi.org/10.1136/neurintsurg2020-016045.

32. Ozdemir O, Giray S, Arlier Z, Baş DF, Inanc Y, Colak E. Predictors of a good outcome after endovascular stroke treatment with stent retrievers. Sci World J 2015;2015:403726. https:/ / doi.org/10.1155/2015/403726.

33. Soize S, Barbe C, Kadziolka K, et al. Predictive factors of outcome and hemorrhage after acute ischemic stroke treated by mechanical thrombectomy with a stentretriever. Neuroradiology 2013;55:977-987. https://doi. org/10.1007/s00234-013-1191-4.

34. Yoon W, Kim SK, Park MS, et al. Predictive factors for good outcome and mortality after stent- retriever thrombectomy in patients with acute anterior circulation stroke. J Stroke 2017;19:97-103 https://doi.org/10.5853/ jos.2016.00675.

35. Dekker L, Geraedts VJ, Hund H, et al. Importance of reperfusion status after intra-arterial thrombectomy for prediction of outcome in anterior circulation large vessel stroke. Intervent Neurol 2018;7:137-147. https:/ / doi.org/ $10.1159 / 000486246$.

36. Flottmann F, Leischner H, Broocks G, et al. Recanalization rate per retrieval attempt in mechanical thrombectomy for acute ischemic stroke. Stroke 2018;49(10):2523-2525. https://doi.org/10.1161/STROKEAHA.118.022737.

37. Goyal M, Menon BK, van Zwam WH, et al. HERMES collaborators. Endovascular thrombectomy after large-vessel ischaemic stroke: a meta-analysis of individual patient data from five randomised trials. Lancet 2016;387:17231731. https: / / doi.org/10.1016/S0140-6736(16)00163-X.

38. Zaidat OO, Castonguay AC, Linfante I, et al. First pass effect: a new measure for stroke thrombectomy devices. Stroke 2018;49:660-666. https://doi.org/10.1161/STROKEAHA.117.020315.

39. Hashimoto T, Hayakawa M, Funatsu N, et al. Histopathologic analysis of retrieved thrombi associated with successful reperfusion after acute stroke thrombectomy. Stroke 2016;47(12):3035-3037. https://doi.org/10.1161/ STROKEAHA.116.015228.

40. Gunning GM, McArdle K, Mirza M, et al. Clot friction variation with fibrin content; implications for resistance to thrombectomy. J Neurointerv Surg 2018;10(1):34-38. https:/ / doi.org/10.1136/neurintsurg-2016-012721.

41. Samaniego EA, Roa JA, Limaye K, Adams Jr HP. Mechanical thrombectomy: emerging technologies and techniques. J Stroke Cerebrovasc Dis 2018;27(10):2555-2571. https:/ / doi. org/10.1016/j.jstrokecerebrovasdis.2018.05.025.

42. Rossi R, Fitzgerald S, Molina S, et al. The administration of rtPA before mechanical thrombectomy in acute ischemic stroke patients is associated with a significant reduction of the retrieved clot area but it does not influence revascularization outcome. J Thromb Thrombolysis 2020. https://doi.org/10.1007/s11239-020-02279-1.

43. Vidale S, Romoli M, Consoli D, Agostoni EC. Bridging versus direct mechanical thrombectomy in acute ischemic stroke: a subgroup pooled meta-analysis for time of intervention, eligibility, and study design. Cerebrovasc Dis 2020;49(2):223-232. https://doi.org/10.1159/000507844.

44. Shah S, Xian Y, Sheng S, et al. Use, temporal trends, and outcomes of endovascular therapy after interhospital transfer in the United States. Circulation 2019;139:15681577. https://doi.org/10.1161/CIRCULATIONAHA.118. 036509.

45. Nogueira RG, Jadhav AP, Haussen DC, et al. Thrombectomy 6 to 24 hours after stroke with a mismatch between deficit and infarct. N Engl J Med 2018;378(1):11-21. https:/ / doi.org/10.1056/NEJMoa1706442.

46. Albers GW, Marks MP, Kemp S, et al. Thrombectomy for stroke at 6 to 16 hours with selection by perfusion imaging. N Engl J Med 2018;378(8):708-718. https://doi.org/ 10.1056/NEJMoa1713973.

47. Saver JL. Time is brain-quantified. Stroke 2006;37 (1):263-266. https:// doi.org/10.1161/01.STR.0000196957. 55928.ab.

48. Gonzalez RG. Imaging-guided acute ischemic stroke therapy: from "time is brain" to "physiology is brain.". AJNR Am J Neuroradiol 2006;27:728-735.

49. Leslie-Mazwi TM, Hamilton S, Mlynash M, et al. Defuse 3 non-dawn patients. Stroke 2019;50(3):618-625. https:// doi.org/10.1161/STROKEAHA.118.023310. 